\title{
Contribution of geophysical methods to the detection of underground cavities in salt series. Development in different projects in Morocco (roads, rail, etc...)
}

\author{
Tlemçani M.K. ${ }^{1}$, Ait Brahim L. ${ }^{2}$, El Mahsani A. ${ }^{3}$ et Labrihmi A. ${ }^{4}$ \\ ${ }^{1,2,3}$ Université Mohammed V, Faculté des \\ sciences, Unité de recherche GEORISK, LGRN, 4 Avenue Ibn Battouta \\ Rabat-Agdal, BP 1014, Rabat, Maroc.
}

\begin{abstract}
As part of the geotechnical studies of a road project, a geophysical investigation by electrical tomography, microgravimetry, and boreholes, was carried out in order to locate if the areas can present or not a risks of ground instability along the road alignment. The road is located near a rock salt mine. This geophysical survey had demonstrated that the area may present major risks for the stability of the road platform. This risk is related to the existence of large expanses of saliferous rocks. The dissolution and exploitation of salt provoked underground voids under the future road. The results of this geophysical survey have allowed the designer office to propose more suitable solutions to solve this problem or even the possibility of shifting the road to another site.
\end{abstract}

\section{Introduction}

Le secteur d'étude est caractérisé en plus de son relief accidenté et difficile par la présence de zones d'effondrement qui sont certainement en relation étroite avec l'activité d'exploitation du sel gemme par la mine qui se trouve dans la région et qui occupe une partie du secteur étudié.

\section{SITUATION ET CONTRAINTES GEOGRAPHIQUES}

Situé à 2,5km au Nord-Ouest de la ville de Taza, dans le domaine rifain, près d'une mine de sel gemme.

Le tronçon routier empreinte le couloir de contact entre deux principaux domaines géologiques, à savoir le domaine atlasique au Sud et le domaine rifain au Nord. Le tronçon traverse des terrains à topographie sinueuse et accidentée, faisant partie du domaine du Prérif externe. Le paysage rencontré est géographiquement plus ou moins homogène et comprend un ensemble de collines généralement de nature marneuse.

\section{CONTEXTE GEOLOGIQUE}

Le site objet de la reconnaissance géophysique fait partie de la zone du contact entre le prérif charrié et l'avant-pays autochtone, $\mathrm{Ce}$ contact frontal contient souvent du gypse associé à du sel gemme sous forme de lits dans la masse charriée (complexe pré rifain). Il s'agit d'un matériel triasique remanié (il est nécessaire d'admettre qu'il a été totalement dissous avant d'être resédimenté (Leblanc, 1979).

Les formations salifères sous forme de lits sont parallèles à la surface de charriage et elles prennent une grande ampleur dans le secteur d'étude formant ainsi le gisement de sel gemme. Ces niveaux évaporitiques sont bien cristallisés et non tectonisés formant des masses irrégulières noyées au sein des sédiments marneux (surtout d'âge Miocène) expliqué aussi par desphénomènes diapiriques contemporains des charriages et qui ont amené le Trias en surface, en provoquant des ruptures dans la couverture tertiaire "figure.1".

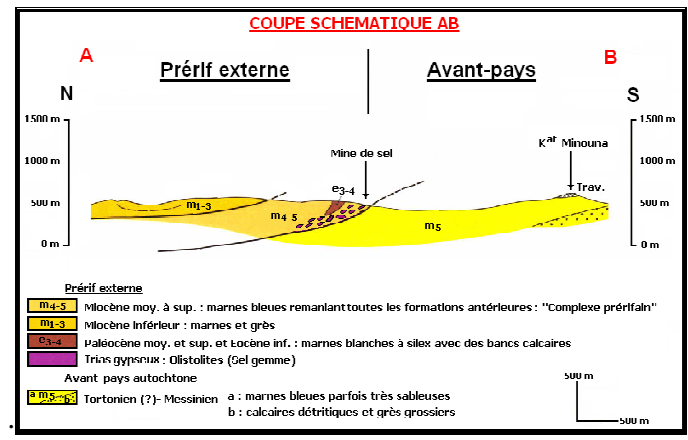

Fig. 1.Coupe géologique $A B$ (Fig. II-7) d'après la carte géologique de Bab El Mrouj Nord Au 1/50 000, 1978

Les tranchées et les galeries creusées par les travaux de la mine montrent généralement trois formations d'épaisseurs variables, à la surface des marnes suivies 
par des intercalations marno-salifères et des masses dures de sel gemme“figure.2".

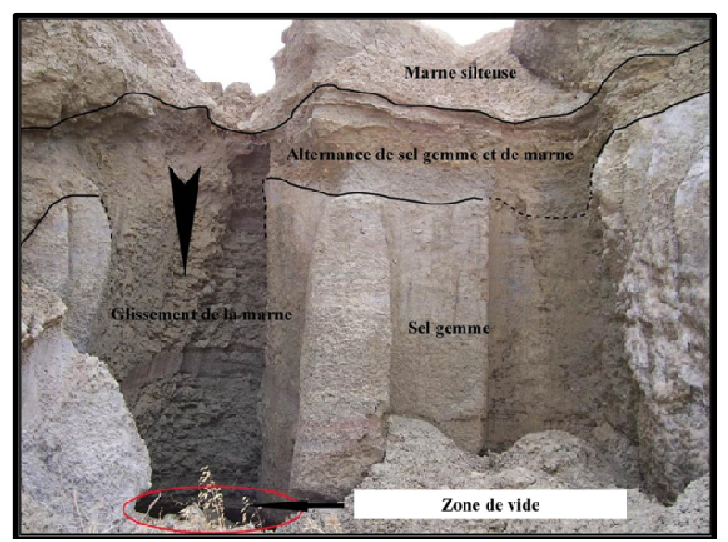

Fig. 2. Dispositif de mesure de tomographie électrique avec quatre câbles

\section{DESCRIPTION DES GEOPHYSIQUE UTILISEES}

\section{1, Tomographie électrique}

La tomographie électrique (ou imagerie) de résistivité électrique est une technique dérivant des méthodes classiques du sondage et du traîné électrique et permettant une imagerie continue 2D ou 3D des variations latérales et verticales des résistivités électriques du sous-sol grâce à des électrodes implantées en surface. Elle permet d'obtenir une coupe de la résistivité en fonction de la profondeur en mesurant le profil de résistivités pour différentes combinaisons d'électrodes de courant et de potentiel "figure.3".

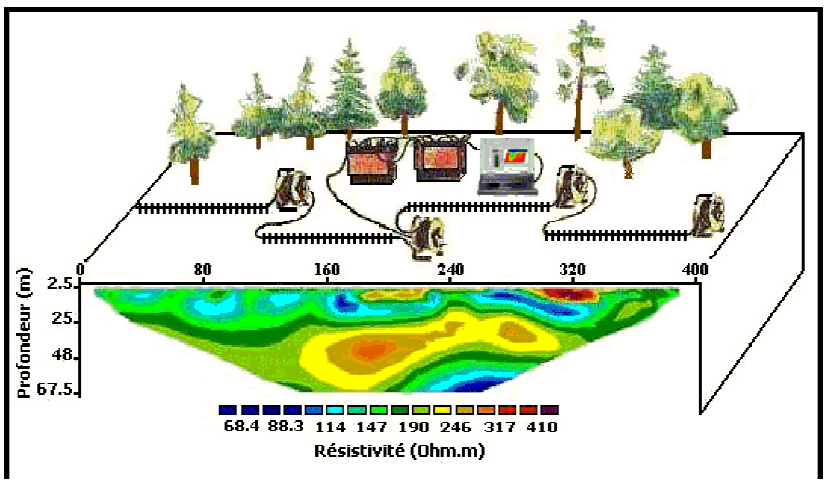

Fig. 3.Dispositif de mesure de tomographie électrique avec quatre câbles

\subsection{La microgravimetrie}

La méthode de prospection micro gravimétrique consiste à mesurer les variations de l'accélération de la pesanteur puis à appliquer aux valeurs brutes un certain nombre de corrections pour éliminer l'influence des autres corps célestes, de la rotation et de la forme de la Terre, afin de ne faire ressortir que les anomalies imputables seulement aux variations de densité du sous- sol. Autrement dit L'analyse des variations de la pesanteur en surface permet d'appréhender la répartition des masses à l'intérieur de la terre à toutes les échelles. L'anomalie de Bouguer est la réponse gravimétrique de toutes les hétérogénéités situées à toutes les profondeurs.

L'anomalie de Bouguer est donnée par la formule suivante:

$\Delta g_{B}=\Delta g_{(\text {mesurée })} \pm$ les 5 corrections

1-Correction de dérive de l'appareil y compris la correction de marée

2- Correction de latitude $\Delta_{\mathrm{L}}=0,081 \sin 2 \varnothing \mathrm{mgals} / 100 \mathrm{~m}$ (Øest latitude en degré).

3- Correction d'altitude $\Delta_{\mathrm{h}}=-0,3086 \mathrm{~h} \mathrm{mgals} / \mathrm{m}$ (h est la côte par apport à la référence)
4- Correction de plated

mgals $/ m\left(\rho_{\mathrm{B}}\right.$ densité de plateau)

5- Correction de terrain $\Delta_{T}$

Où

$\Delta \mathrm{g}_{\text {(mesurée) }}=\mathrm{g}_{\text {mesurée }}-\mathrm{g}_{\text {th }}$

Donc

$\Delta \mathrm{g}_{\mathrm{B}}=\mathrm{g}$ mesurée $-\left(\mathrm{g}_{\text {th }} \pm \Delta \mathrm{g}\right)$

Avec:g mesurée pesanteur mesurée $\mathrm{g}_{\text {th }}$ la pesanteur théorique : $\mathrm{g}_{\mathrm{th}}=978.0490\left(1+0.0052884 \sin ^{2} \varnothing-0.0000059 \sin ^{2} 2 \varnothing\right) \mathrm{en}$ gal

L'anomalie de Bouguer est représentée sous forme de carte dite de l'anomalie de Bouguer qui est le document de base en gravimétrie.

\section{PROSPECTION GEOPHYSIQUE}

\section{1, Présentation}

La campagne géophysique, avait comme objectifs :

- La délimitation des horizons salifères ;

- La détection des cavités souterraines crées par l'exploitation du minerai le long de l'axe routier ;

- La localisation des points singuliers et des zones pouvant présenter des risques d'instabilité de terrain.

$\mathrm{Au}$ total, six (6) profils tomographiques parallèles ont été réalisés avec des longueurs de $295 \mathrm{~m}$. Chaque profil est réalisé avec deux câbles colinéaires et formé par 60 électrodes avec un espacement constant de 5m. Les profils sont orientés sensiblement SW-NE avec une distance variable entre eux, elle est de l'ordre de $40 \mathrm{~m}$ entre les profils successifs $1,2,3$ et 4 et de l'ordre de $30 \mathrm{~m}$ entre les profils 4,5 et 6 .

Les mêmes profils tomographiques sont exploités pour les mesures microgravimétriques; l'emplacement de 
chaque électrode est pris comme une station microgravimétrique en ajoutant entre deux électrodes successives une station (donc 2,5m entre deux stations voisines appartenant au même profil). Au total, 720 stations de mesure microgravimétrique réparties sur 6 profils avec 120 stations par profil.

L'ensemble de toutes les stations de mesures tomographiques et microgravimétriques ainsi que les sondages mécaniques exécutés sont présenté sur la "figure.4".

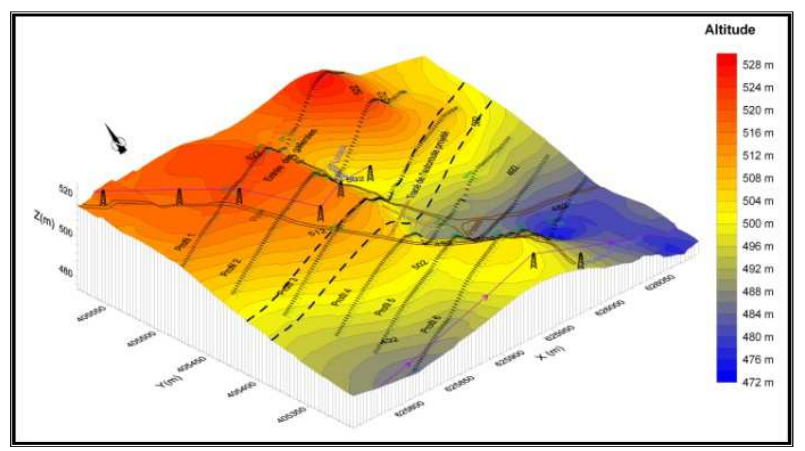

Fig.4.Carte de situation des profils de tomographieélectrique,microgravimétrique, et des sondages carottés par rapport au tracé de l'autoroute en mode 3D

\subsection{Discussion des résultats}

\section{$\checkmark \quad$ Tomographie électrique}

Les résultats après inversion des données de résistivité apparente donnent des images électriques de résistivités vraies du sous sol et de profondeurs, ce qui permet une interprétation quantitative des données. Mais ces images restent insuffisantes et l'espace des solutions demeure assez vaste. Cependant les divers relevés d'affleurements et les caractéristiques géologiques du terrain le long des profils ont permis de préciser et d'interpréter les images électriques obtenues du sous-sol.

On a systématiquement observé, sur chacune des images électriques, la présence de zones très conductrices dont la résistivité est très faible (représentées en bleu sur toutes les images électriques) et dont l'extension latéraleet verticale est variable. Ces zones, faiblement résistantes correspondent avec forte probabilité aux niveaux marneux. Le niveau représentant l'alternance des marnes et du sel (représenté en tranches vert-marron) apparaît dans les images électriques comme une plage de valeurs moyennes de résistivité. Ce niveau est aussi caractérisé par une extension variable latéralement et verticalement.

Les zones d'anomalies de résistivité (représentées par des «noyaux» rouges-violets sont généralement localisées entre 150 et $170 \mathrm{~m}$ mais avec des profondeurs variables qui restent généralement dans les quinze premiers mètres, ces zones présentent aussi des variations latérales importantes ; elles sont largement allongées sous les profils $1,2,3$ et 5 et très réduites sous les profils 4 et 6. A partir de ces résultats, nous pouvons admettre que les zones résistantes forment une continuité du profil 1 au profil 6 et que son extension verticale et latérale s'est avérée variable. Les indices géologiques et les relevés d'affleurement permettent de rattacher la zone d'anomalie au niveau du profil 6 aux masses de sel gemme. Les autres zones sont rattachées soit aux masses de sel gemme ou aux cavités. Cette interprétation reste dans le rang d'une hypothèse qui sollicite une confirmation par une autre méthode géophysique, à savoir la microgravimétrie"figure.5".
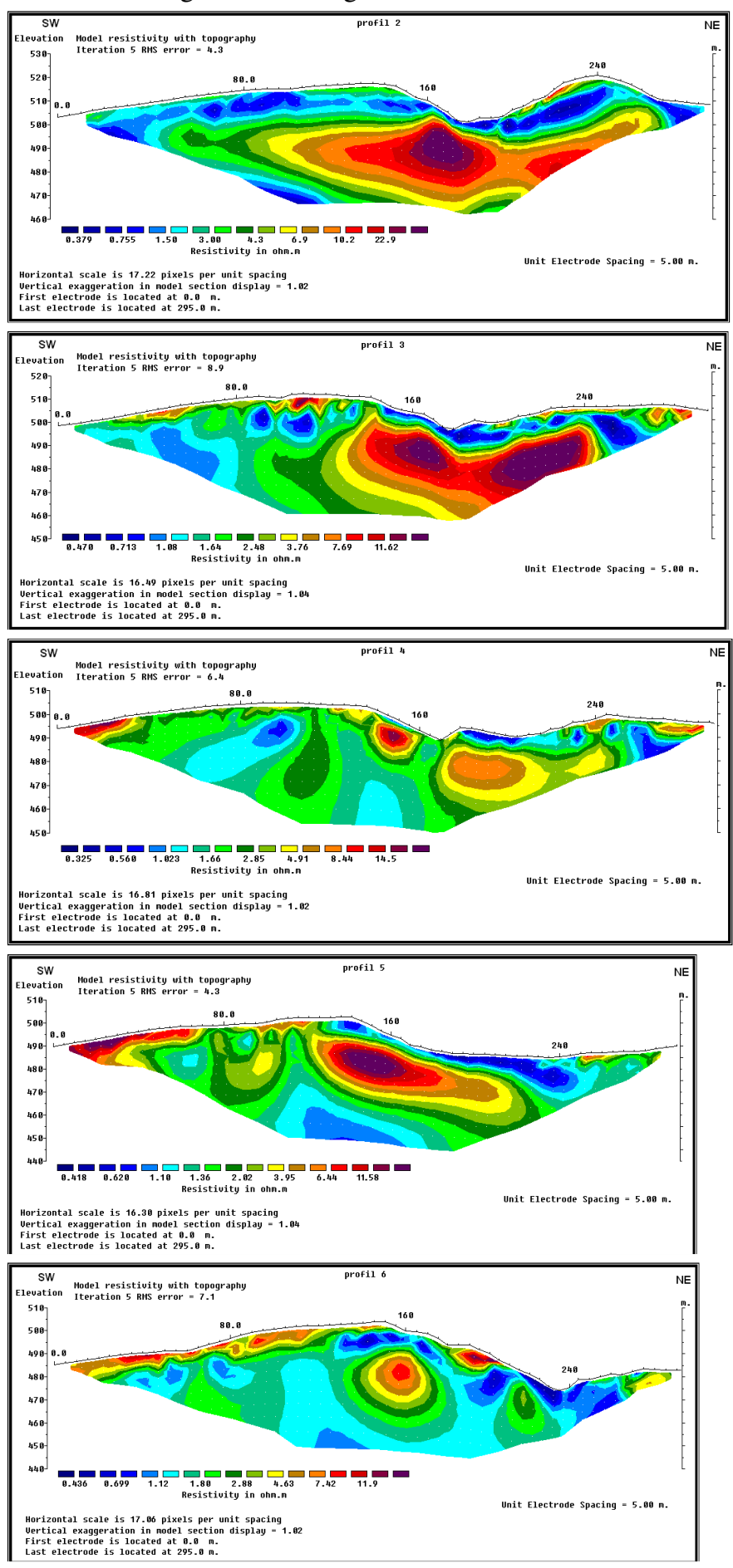

Fig.5. Modèle de résistivité électrique obtenu par inversion 2D des données des profils

\section{3, Synthèse des résultats}

\subsection{1, La microgravimétrie}


La campagne de microgravimétrie vise à confirmer les résultats de la tomographie électrique d'une part, et d'autre part, de répondre le mieux possible aux hypothèses déjà posées en ce qui concerne la présence ou non des cavités et des masses de sel gemme

Les mesures ont été effectuées à l'aide d'un gravimètre Scintrex CG5.Une large gamme de densités a été employée pour comparer les déférentes cartes d'anomalie de Bouguer qui en résultent, afin de choisir la carte qui présente le mieux le contraste en terme de densité (entre les marnes, le sel gemme et/ou les cavités) et donc qui permet au mieux de révéler les zones d'anomalies.

La carte d'anomalie de Bouguer pour les densités $2.0 \mathrm{~g} / \mathrm{cm} 3$ "figure.6" montre un bon contraste et par conséquent elles dévoilent certaines anomalies locales; on cite particulièrement les anomalies négatives au niveau du profil 1 (de station 1068 à 1075), du profil 2 (de station 2072 à 2073 et de station 2094 à 2099), du profil 4 (de station 4092 à 4095 et de station 4088 à 4091) et du profil 6 (de station 6092 à 6098).

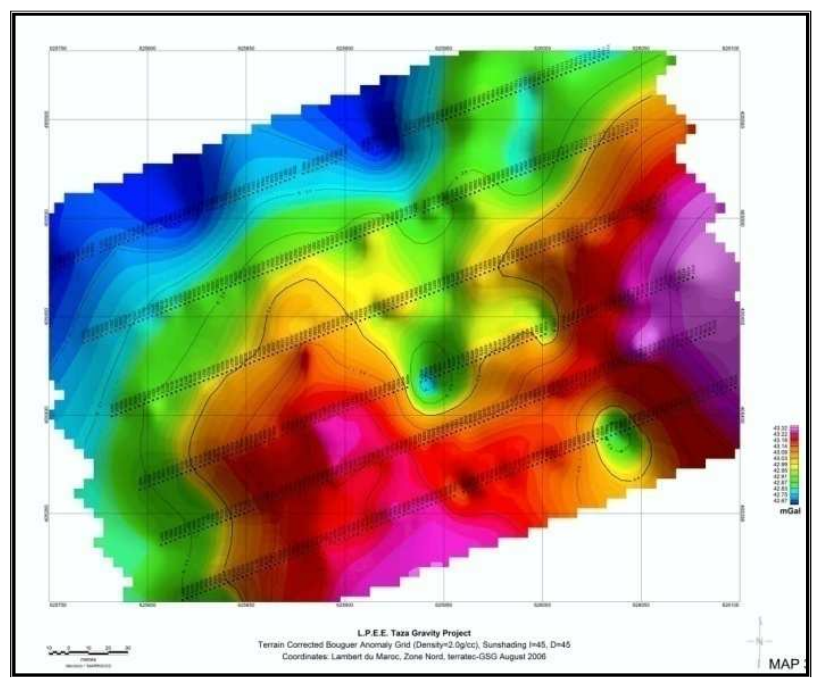

Fig.6.Carte d'anomalie de Bouguer (densité $=2,0 \mathrm{~g} / \mathrm{cm} 3$ )

Pour une meilleure analyse de cette carte on procède

àlatechnique de calcul de la dérivée première $\left(\delta \Delta g_{B} / \delta z\right.$ ) dite aussi le gradient vertical. Cette technique permet de réduire l'effet de coalescence des sources d'anomalies et d'amplifier les anomalies dues aux structures superficielles par rapport à celles liées aux structures profondes. L'application de cette technique permet d'élaborer la carte de la première dérivée de l'anomalie de Bouguer "figure.8". L'analyse de cette carte montre clairement des anomalies (zones bleues) qui s'individualisent par endroits sur tous les profils.

Toutes ces cartes traitées indiquent la présence du sel gemme de faible densité au Nord et à l'Ouest et des sédiments marneux de densité plus élevée au Sud-Est du secteur d'étude. On observe en plus un certain nombre d'anomalies sur la carte du gradient verticale qui montrent de possibles cavités dans le sel. D'autres anomalies semblent être associées aux effets de terrain ceci est justifié par le fait que la correction de terrain est basée sur le modèle numérique de terrain qui n'a pas été assez précis pour se débarrasser de tous ces effets. Des annotations sur les figures 6 et 7 ont été faites pour montrer les interprétations adoptées.

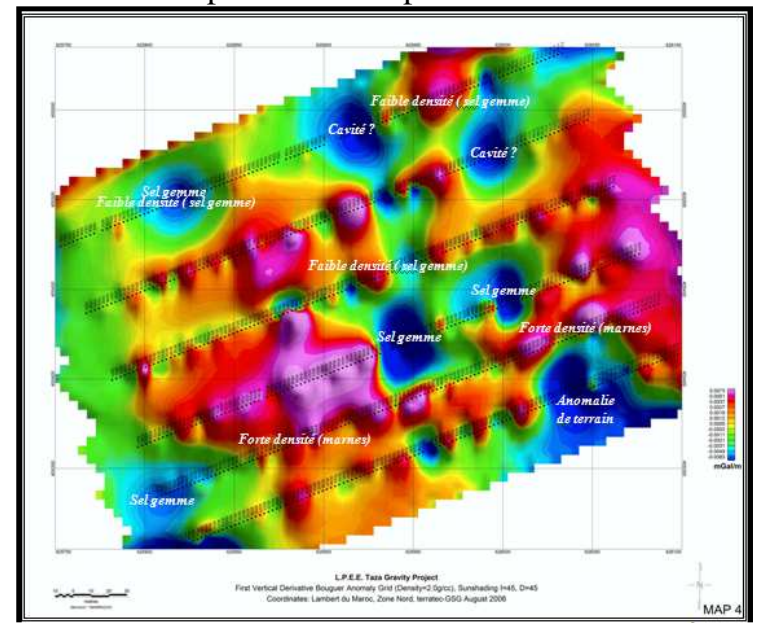

Fig.7.Carte de la première dérivée de l'anomalie de Bouguer (densité $=2,0 \mathrm{~g} / \mathrm{cm} 3$ )

\subsubsection{Les sondages carottés}

$\mathrm{Au}$ total 11 sondages carottés de $30 \mathrm{~m}$ de profondeur ont été exécutés et qui sont répartis sur tous les profils de tomographie électrique et de microgravimétrie. Ces sondages sont exécutés pour caler les résultats de la campagne géophysique et de vérifier les interprétations adoptées a fin de juger un certain nombre d'anomalies Les coupes lithologiques des sondages carottés exécutés confirment la vision lithologique concernant la répartition des formations géologiques dans le secteur d'étude. Ils montrent formellement que les terrains de recouvrement sont essentiellement marneux (et par endroits des masses de sel gemme en affleurement) qui reposent généralement sur une alternance marno-salifères et en profondeur on trouve généralement des marnes grisâtres. Ces coupes montrent également qu'il y a une importante variation latérale et verticale des formations géologiques dans le secteur étudié.Les résultats de ces sondages sont représentés dans les figures suivantes "figure.8,9,10,11,12,13":

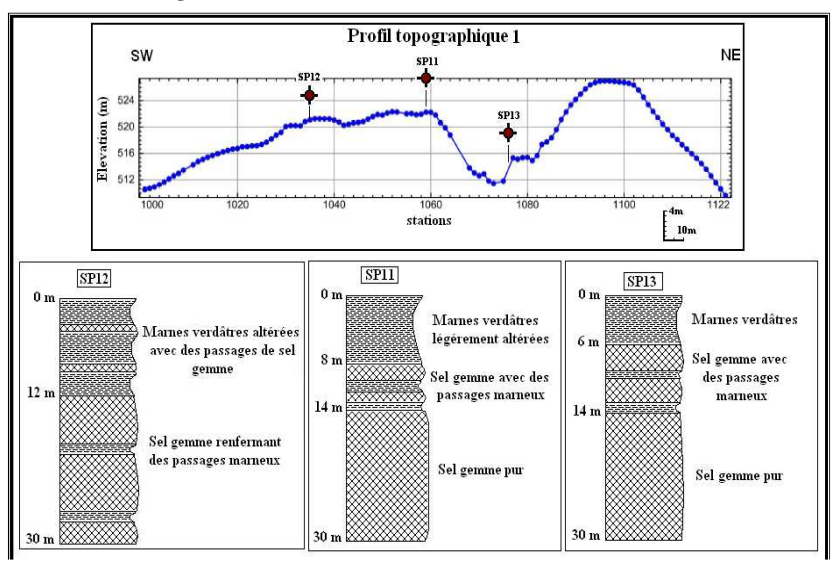

Fig.8. Coupes lithologiques des sondages carottés SP11, SP12 et SP13, leurs emplacements par rapport aux stations gravimétriques, et topographie du profil 1 . 


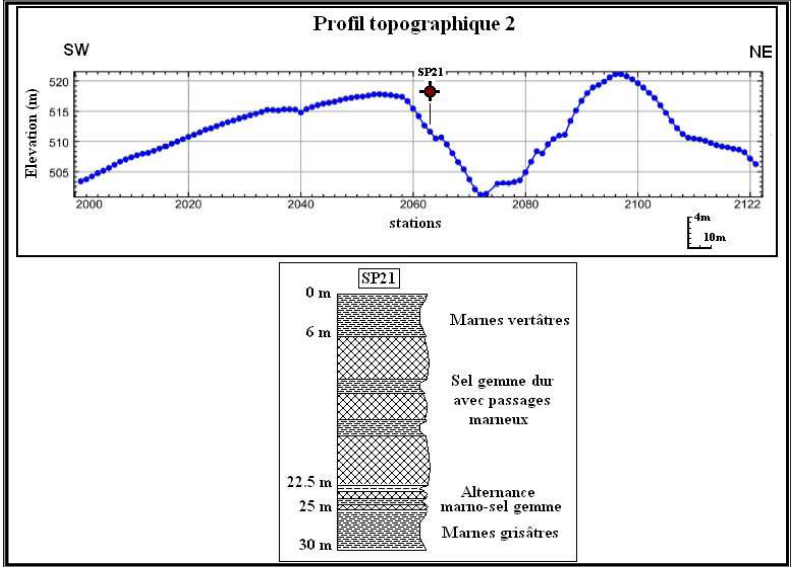

Fig.9.Coupe lithologique du sondage carotté SP21, son emplacement par rapport aux stations gravimétriques, et topographie du profil 2

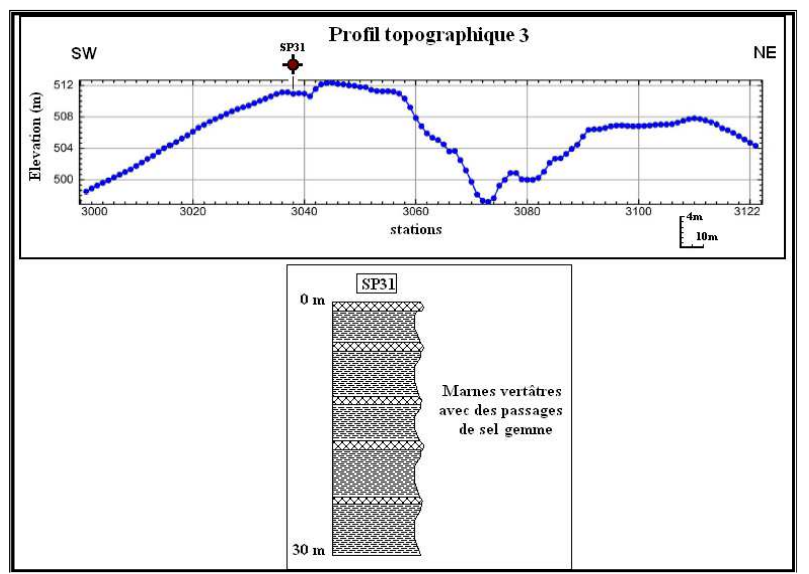

Fig.10.Coupe lithologique du sondage carotté SP31, son emplacement par rapport aux stations gravimétriques, et topographie du profil 3

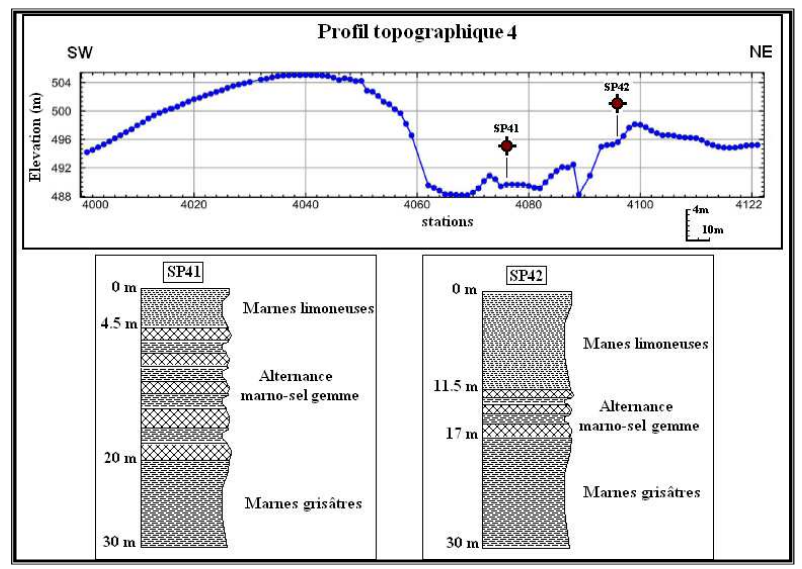

Fig.11.Coupes lithologiques des sondages carottés SP41 etSP42, leurs emplacements par rapport aux stationsgravimétriques, et topographie du profil 4

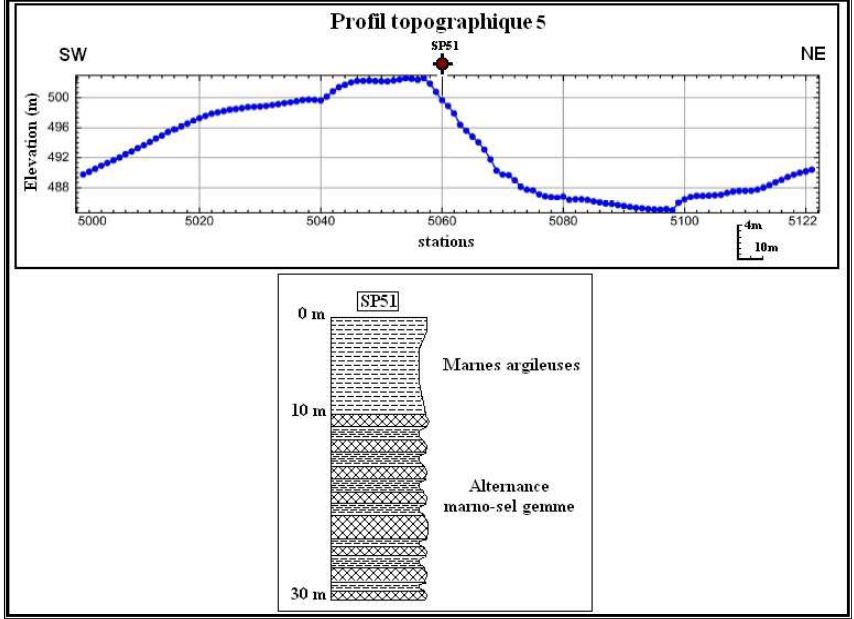

Fig.12.Coupe lithologique du sondage carotté SP51, son emplacement par rapport aux stations gravimétriques, et topographie du profil 5.

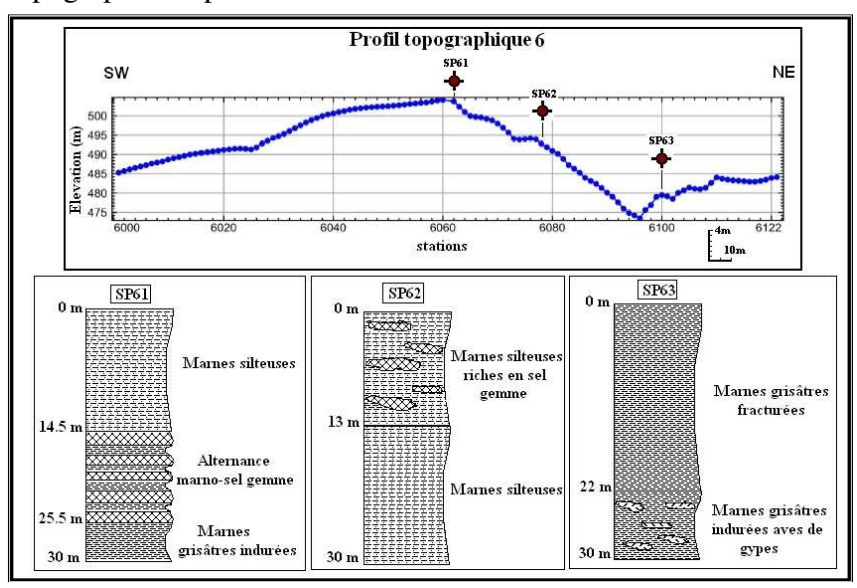

Fig.13.Coupes lithologiques des sondages carottés SP61, SP62 et SP63, leurs emplacements par rapport aux stations gravimétriques, et topographie du profil 6 .

\section{SYNTHESE GENERALE DES RESULTATS}

A la lumière des données de la prospection de la tomographie électrique et de la microgravimétrie couplées par des sondages carottés, il a été possibled'élaborermodèle géologique de terrain faisant sortir les anomalies pouvant être en relation avec les cavités.

L'examen de la carte de la dérivée première de l'anomalie de Bouguer révèle l'existence de plusieurs anomalies positives interprétées comme formations marneuses et négatives interprétées par l'existence de masses de sel gemme et/ou de cavités. Ces anomalies sont bien corrélées aux résultats de tomographie électrique du secteur étudié. Leur confrontation aux données géologiques a permis de faire les observations suivantes :

- Seules les anomalies de résistivité et de densité au niveau des profils 1 et 2 semblent être des zones de vides possibles puisqu'elles présentent des valeurs de résistivités elevés par rapport aux autres anomalies des autres profils. En plus, les sondages mécaniques SP11, 
SP13 et SP21 ne touchent pas le centre des anomalies, ils sont juste passés par la périphérie de ces anomalies. Ces sondages montrent aussi qu'il y'a des vastes masses de sel gemme.

- La carte de la première dérivée de l'anomalie de Bouguer ne montre pas des anomalies bien visibles au niveau de la ligne gravimétrique 3 , mais le profil 3 de tomographie électrique montre qu'il y a une importante anomalie mais avec de valeurs de résistivités inférieures à celles rencontrées au niveau des profils 1 et 2 . Pour ces raisons on a interprété cette zone comme une zone de concentration de sel gemme éliminant ainsi la possibilité d'existence de vides.

- La carte de la première dérivée de l'anomalie de Bouguer montre clairement des anomalies gravimétriques au niveau des profils 4,5 et 6 ce qui est confirmé aussi au niveau des profils de tomographie électrique par des zones de résistivités peu élevées. Ces zones d'anomalies font l'objet des sondages mécaniques SP41, SP42, SP51, SP61 et SP62. Ces derniers montrent qu'il s'agit uniquement d'une concentration de sel gemme.

- Enfin, la zone d'anomalie gravimétrique localisée à l'extrémité du profil 6 ne trouve pas sa ressemblance en termes de résistivité par la tomographie électrique. Le sondage carotté SP63 montre qu'il s'agit de formations marneuses ce qui est concordant avec les valeurs de résistivités obtenues. L'anomalie gravimétrique est attribuée donc aux effets de terrain c'est-à-dire à des corrections de terrains qui probablement ne sont pas bien faites.

\section{CONCLUSION GENERALE}

La prospection géophysique utilisant la méthode de tomographie électrique et la microgravimétrie pour la reconnaissance du sous sol du tracé d'un projet routier près d'une mine de sel gemme a permis de mettre en évidence des zones d'anomalies de résistivité et de densité. Ces zones sont corrélées avec les sondages carottés, les conditions géologiques et avec les relevés d'affleurement du terrain afin de les interpréter. Cette corrélation a permis de délimiter d'importantes formations salifères et de désigner quelques zones qui peuvent contenir des vides.

Le couplage des données de la géophysique et des autres levés (sondages carottés, données géologiques) s'est donc avéré efficace, aboutissant ainsi à un modèle géologique satisfaisant. Cependant les résultats de cette prospection sont nettement défavorables pour le passage de tracée routier, car en plus du relief accentué, on note l'existence possible de cavités qui présentent des menaces d'effondrement et l'existence de vastes masses de sel gemme qui présentent des dangers non négligeables liés à leur dissolution en présence de l'eau et donc un risque d'instabilité de terrain.

En se basant sur ces résultats, le secteur étudié est jugé non valable pour le passage de la route et il est préconisé de trouver une autre variante vers le Sud du terrain prospecté où les masses de sel gemme sont moins importantes.

\section{REFERENCES BIBLIOGRAPHIQUES}

1. M.A. Annecchione, La contribution de la gravimétrie à l'étude hydrogéologique de la Moraine OakRidges, mémoire de maîtrise ès sciences appliquées (génie minéral), Ecole Polytechnique de Montréal, 124p (2004).

2. R.D. Barker, Depth of investigation of collinear symmetrical four- electrode arrays, Geophysics, 54(8):1031-1037 (1989).

3. G. Bernard, Techniques géophysiques de haute résolution, Microgravimétrie, cours en ligne, Ecole Polytechnique de Montréal, 48p (2005).

4. http://geo.polymtl.ca/ giroux/cours/gml6201a/docu ments/gravi.pdf

5. G. Bernard, Techniques géophysiques de haute résolution, Tomographie géophysique en forage, cours en ligne, Ecole Polytechnique de Montréal, 47p (2005).

6. M. Chouteau, Géophysique Appliquée I, gravimétrie, cours en ligne, Ecole Polytechnique de Montréal, 108p (2002).

7. http://geo.polymtl.ca/www_Cours/pdffiles/Gravimetr ie.pdf

8. M. Chouteau. et E.Gloaguen, Tomographie électrique en génie et en environnement, cours de tomographie électrique, 64p (2003).

9. http://geo.polymtl.ca/ chouteau/documents/GML620 1_tomoelec.ppt

10. M. Combe, Le couloir de Fès-Taza (les ressources en eau du Maroc Tomme 2), Notes et Mémoires du service géologique $\mathrm{n}^{\circ} 231$ Rabat (1975).

11. I D'amours, Modélisation et interprétation de tomographie de résistivité et de polarisation provoquée entre trous de forage, mémoire de maître ès sciences appliquées (Génie Minéral), Ecole polytechnique de Montréal, 156p (1998).

12. A Dridri. et B.Fedan, Rôle du contrôle structural dans la mise en place du réseau hydrographique de Sebou et d'Inaouène entre Fès et Oued Amlil, Bulletin de l'Institut scientifique, Rabat, Section sciences de la Terre, $\mathrm{n}^{\circ 23}$, 10p, pp 67-77 (2001).

13. http://www.israbat.ac.ma/PublicationsIS/BIS_ST_23 $\% 5$ Cdridri_morpho.pdf.

14. L.S. Edwaerds, A modified pseudosection for resistively and induced-polarization. Geophysics, 42p (1977).

15. B. El hassani, Etude hydrogéologique du couloir FèsTaza, Mémoire de fin d'études, ENIM, Rabat, 116p (1994).

16. A. Gartet, J.Gartet. et CC García C. C., Exploitation des salines au massif diapirique de tissa et impacts. 